3. Noda, M. et al. Nature 305, 818-823 (1983).

4. Giraudat. J. et al. Proc. nam. Acad. Sci. U.S.A. 83, $2719-$ $2723(1986)$

5. Oberthür. W. et al. EMBOJ. 5, 1815-1819 (1986)

6. Imoto. K. et al. Nature 324, 670-674 (1986).

7. Finer-Moore. J. \& Stroud. R.M. Proc. nutn. Acad. Sci. U.S.A. 81. 155.159(1984).

8. Barnard. E.A.. Darlison. M.G. \& Seeburg. P.H. Trends Neurosci. 10, 502-509 (1987)

9. Hille. B. Ionic Channels of Excitable Membranes (Sinauer Sunderland. Massachusets, 1984).

\section{Aperiodicity of magnetic reversals?}

SIR-Recently, Nature has published another analysis of data on the Earth's magnetic reversals where a 'statistically significant period' of $\sim$ of $30 \mathrm{Myr}$ is claimed to have been demonstrated'. Unfortunately, the statistical argument supporting this conclusion is flawed; the test performed could equally well be taken as demonstrating any other period or no period at all. The argument for significance given by Stothers is based on an incorrect use of a correlation coefficient to compare two columns of figures; the relevant portion of that author's Table 2 is reproduced in the first two columns of the table below. These columns give the peaks of spectra for the 'observed' magnetic reversal series and for an 'ideal' series of events equally spaced 30.1 Myr apart.

$\begin{array}{ccc}\begin{array}{c}\text { Observed } \\ \text { series }\end{array} & \begin{array}{c}\text { Ideal } \\ \text { series }\end{array} & \begin{array}{c}\text { Rescaled (by 0.8) } \\ \text { peaks }\end{array} \\ 48.2 & 48.0 & 38.4 \\ 38.0 & 40.5 & 32.4 \\ 30.1 & 30.1 & 24.08 \\ 25.2 & 24.0 & 19.2 \\ 21.9 & 20.7 & 16.56 \\ 19.4 & 19.5 & 15.6 \\ 17.2 & 17.3 & 13.84 \\ 15.5 & 15.1 & 12.08 \\ 13.4 & 13.1 & 10.48 \\ 12.0 & 12.0 & 9.6 \\ 10.9 & 11.0 & 8.8 \\ 10.0 & 10.0 & 8.0\end{array}$

To quantify the appearance of agreement between columns one and two, Stothers calculated their correlation as $r=0.997$, and this is the basis for the claimed statistical significance of the period of $30.1 \mathrm{Myr}$. (Actually, he omits the pair $(30.1,30.1)$ from the computation. This affects neither his result nor the point made here.)

The third column given here is formed by multiplying column two by 0.8 ; it thus represents peaks in the spectrum for an ideal series of events equally spaced by $30.1 \times 0.8=24.08 \mathrm{Myr}$. Of course, one of the properties of the correlation coefficient is that it is unchanged by linear rescaling, so the correlation of columns one and three is also $r=0.997$, and, if we accept the argument as presented, we are led to conclude that there is also a 'statistically significant period' of $24.08 \mathrm{Myr}$. In fact, the number 0.8 is arbitrary; can the magnetic reversal series really be in 'statistically significant' agreement with any (and all) possible periods?

The basic problem is that the correla- tion coefficient is an inappropriate device for comparing two monotone sequences - any two monotone sequences will appear to be highly correlated. The author has tried to allow for this. but the attempt has misfired. To assess significance he has compared his value 0.997 to what is an irrelevant set of reference values: 5,000 sets of eleven numbers were drawn independently and uniformly from the range $10-58$ and placed in increasing order; their correlations with the observed series (omitting 30.1) served as the reference set, and in comparison with this set the value 0.997 does seem extreme. But peaks of spectra of random series do not behave like uniformly distributed random variables, whether they are periodic or not. The peaks exhibit patterns of the type the author's Figure 2 displays: clumping at low values and increasing sparsity at large values. If the author had referred his correlation coefficient to a set of values generated in some reasonable way from spectra of random series, the test might have been of some interest (although as a test of general periodicity, not of a 30.1 Myr period, because of the correlation coefficient's insensitivity to rescaling). As it is, the test is utterly uninformative about the stochastic behaviour of magnetic reversals. As Stothers notes, the other test he performed to bolster the claim of statistical significance is questionable on other grounds (it allows for neither the nonstationary nature of the series nor the $a$ posteriori selection of $30 \mathrm{Myr}$ as a period of interest). The question of the existence of a period in these data remains open.

Department of Statistics,

Stephen M. Stigler

University of Chicago,

5734 University Avenue,

Chicago, Illinois 60637, USA

1. Stothers, R. B. Nature $322,444-446$ (1986)

Stothers REPLIES - Stigler criticizes two aspects of my formal tests for statistical significance of an apparent 30-Myr periodicity in the Earth's magnetic reversals'. First, he argues that, as the correlation coefficient between any two sequences of numbers is independent of whatever constant factor multiplies each sequence, the sequence of spectral-peak periods derived for a perfectly periodic (ideal) time series can be arbitrarily multiplied by a constant factor and compared with the analogous sequence derived from an observed time series. Thus, any choice of generating period for the ideal time series must give rise to the same value of the correlation coefficient.

Stigler's argument is largely incorrect for the following reason. Consider the harmonic structure, rather than the absolute values, of the spectral-peak periods. (The high peaks are quite obvious in the spectra; the contrast between them and all lower peaks is sufficiently large so as not to require any special criteria to distinguish them.) To cover the same range of (dimensionless) periods in the observed and ideal spectra, the periods of the peaks must be normalized so that the generating period is formally equal to the assumed basic observational period. But a different choice of generating period necessarily implies a different number of cycles covered in a time series of fixed length. This change affects both the number and the relative period distribution of the spectral peaks. For example, if the generating period is taken to be (dimensionally) $15.5 \mathrm{Myr}$, which corresponds to one of the major peaks in the observed spectrum and happens to be the basic period advocated by Mazaud et al. ${ }^{2}$, the ideal time series covering the same length of record, 165 Myr, must contain 11 dates (as compared to six dates if the generating period is 30.1 Myr). The spectrum for the ideal time series in this case consists of 14 high peaks over the period range $10-58 \mathrm{Myr}$, instead of the 12 high peaks obtained with a generating period of $30.1 \mathrm{Myr}$. The spectrum for the observed time series shows 12 high peaks, whose number and relative period distribution (period ratios) are in close accordance with those for a generating period of $30.1 \mathrm{Myr}$ (ref. 1). The dimensional value of the generating period does matter fundamentally, of course, because the physical units in the problem set the dimensions of all periods. Insofar as any other choice of generating period leads to a round number of six dates in the ideal time series, Stigler's criticism is valid, however. This means that the true generating period may lie anywhere in the range $28-33 \mathrm{Myr}$. But if the question is simply whether $30.1 \mathrm{Myr}$, in its role as an actual period of one of the high spectral peaks in the observed time series, is the generating period for the other peaks, the answer is yes; the periods of the other peaks will not work as generating periods.

The second objection raised by Stigler is that even for a random time series the distribution of periods of the high spectral peaks is not completely random but is strongly biased toward shorter periods. $\mathrm{He}$ is correct here. His suggested remedy, however, is not practicable because not all random time series produce exactly 12 high spectral peaks over the fixed period range 10-58 Myr and because rejection of nonconforming cases would make the test less random. A practical approach is my original test ${ }^{\prime}$, but using 12 ordered but randomly generated frequencies (rather than periods), because the frequency distribution of spectral peaks for both random and ideal (as well as observed) time series turns out, from inspection of many cases, to be roughly uniform (statistically) over the frequency range in question. The occasional generation of two close frequencies in a random set of fre- 